\title{
Posicionamento estratégico, estratégias de manufatura e gestão de custos: estudo de caso em uma empresa do segmento metalmecânico
}

Strategic positioning, manufacturing strategies and cost management: case study in a company of metal mechanic segment

Posicionamiento estratégico, estrategias de fabricación y administración de costos: un estudio de caso sobre la empresa de segmento metal mecánica

\section{Fabio Antonio Sartori Piran}

Mestre em Engenharia de Produção e Sistemas pela Universidade do Vale dos Sinos

Professor Assistente na Universidade FEEVALE

Endereço: ERS 239, n॰ 2755, Instituto de Ciências Exatas e Tecnológicas, Bairro Vila Nova

CEP: 93525-075 - Novo Hamburgo /RS - Brasil

E-mail: fabiopiran@feevale.br

Telefone: + 55 (51) 3586-8800

\section{Adão Alberto Blanco Nunes}

Mestre em Ciências Contábeis pela Faculdade de Ciências Contábeis da Universidade do Vale do Rio dos Sinos (Unisinos)

Professor da Faculdade Porto Alegrense (FAPA)

Endereço: Avenida Manoel Elias, n॰ 2.001, Morro Santana

CEP: 91240-261 - Porto Alegre/RS - Brasil

E-mail: albertoblanco.n@bol.com.br

Fone: + 55 (51)3382-8282

\section{Marcos Antonio de Souza}

Doutor em Controladoria e Contabilidade pela Universidade de São Paulo

Endereço: Av. Unisinos, n॰ 950, Econômicas, Sala 5A408, Bairro Cristo Rei

CEP: 93022-000 - São Leopoldo/RS - Brasil

E-mail:marcosas@unisinos.br

Telefone: + 55 (51) 3037-1000, Ramal 1569 ou + (55) (51) 8246-6000

\section{Fabiano de Lima Nunes}

Mestre em Engenharia de Produção e Sistemas pela Universidade do Vale dos Sinos

Professor Assistente na Universidade FEEVALE

Endereço: ERS 239, n॰ 2755, Instituto de Ciências Sociais Aplicadas, Bairro Vila Nova

CEP: 93525-075 - Novo Hamburgo/RS - Brasil

E-mail: fabianonunes@feevale.br

Telefone: + 55 (51) 3586-8800

Artigo recebido em 24/02/2016. Revisado por pares em 30/03/2016. Reformulado em 20/04/2016. Recomendado para publicação em 16/05/2016 por Sandra Rolim Ensslin (Editora Científica). Publicado em 14/06/2016. 


\title{
Resumo
}

O objetivo deste artigo é analisar como a gestão de custos apoia a integração entre posicionamento estratégico e estratégia de manufatura, em uma subsidiária brasileira de uma empresa alemã, que atua no segmento metal mecânico. Trata-se de um estudo de caso único desenvolvido em uma empresa multinacional, com filial no estado do Rio Grande do Sul. Para coleta de dados foram realizadas entrevistas, análise documental e observação direta na organização objeto de estudo. Dentre os principais resultados constatou-se que a empresa compete na estratégia de diferenciação na sua principal linha de produtos, e existe forte competição por liderança de custos entre as subsidiárias da organização, o que sugere características da estratégia híbrida. A empresa adotou a estratégia de manufatura enxuta e fábricas focalizadas, e utiliza a gestão de custos como principal ferramenta para gerenciamento das melhorias realizadas. Também utiliza o custeio padrão, meta e kaizen para controle gerencial.

Palavras-chave: Posicionamento estratégico. Estratégias de manufatura. Gestão de custos.

\begin{abstract}
The objective of this article is to examine how cost management can support the integration of strategic positioning and manufacturing strategy in a company engaged in the metal mechanic segment. This is a single case study with a qualitative and descriptive approach developed in a multinational company with a branch in the state of Rio Grande do Sul. The data collection company was realized with interviews, document analysis and direct observation in the organization object of study. Among the main results we found that the company competes in the differentiation strategy in its main product line, and there is a strong competition for cost leadership among the subsidiaries of the organization, suggesting characteristics of hybrid strategy. The company adopted the strategy of lean manufacturing and focused factories, and using cost management as the primary tool for managing these improvements. Also uses standard costing, target and kaizen for management control.
\end{abstract}

Keywords: Strategic positioning. Manufacturing strategies. Cost management.

\section{Resumen}

El propósito de este artículo es analizar cómo la gestión de costos apoya la integración entre el posicionamiento estratégico y la estrategia de fabricación de una filial brasileña de una empresa alemana dedicada a la serie de sesiones de metal mecánica. Se trata de un estudio de caso único convertido en una empresa multinacional con una rama en el estado de Rio Grande do Sul. Para se llevaron a cabo entrevistas de recopilación de datos, análisis de documentos y observación directa en la organización estudiada. Entre los principales resultados se encontró que la empresa compite en la estrategia de diferenciación en su línea de productos principales, y existe una fuerte competencia por el lidérense de costos entre las filiales de la organización, lo que sugiere característica de la estrategia híbrida. La empresa adoptó la estrategia de eficiencia en la fabricación y las fábricas enfocados, y utiliza los costes de gestión como la principal herramienta para la gestión de las mejoras. También utiliza el costeo estándar, objetivo y kaizen para el control de la gestión.

Palabras clave: Posicionamiento estratégico. Estrategias de fabricación. Gestión estratégica de costos

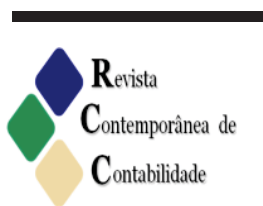




\section{Introdução}

Os efeitos da globalização dos mercados, entre outros fatores, exigem maior ênfase no planejamento, execução e controle das estratégias organizacionais. O estudo do ambiente externo (mercado) e interno (empresa) é destacado por Yarbrough, Morgan e Vorhies (2011) como relevante para que uma organização defina seu posicionamento estratégico e sua estratégia de manufatura. Ortega, Vega e Machuca (2012) mencionam que a estrutura de manufatura deve ser capaz de proporcionar suporte apropriado à estratégia de negócios da organização.

Pacheco e Calarge (2012) citam que, para conseguir ou manter as vantagens competitivas, é preciso que as organizações busquem integrar fatores como estratégias e gestão de custos. Para Cavalcanti, Ferreira e Araujo (2013), a busca pela melhoria contínua dos resultados dos recursos aplicados nos processos produtivos deu ênfase à preocupação das organizações com seus custos, levando-as a utilizar, estrategicamente, as informações geradas pela gestão de custos no seu processo decisório.

Com base neste contexto, o objetivo deste artigo é analisar como a gestão de custos apoia a integração entre posicionamento estratégico e estratégia de manufatura, em uma subsidiária brasileira de uma empresa alemã, que atua no segmento metalmecânico.

Neste sentido, a justificativa para elaboração deste trabalho é contribuir com a discussão presente na literatura sobre as relações dos temas posicionamento estratégico e estratégias de produção (THUN, 2008; MILTENBURG, 2008; DUPONT, 2011; HAN; PORTERFIELD; LI, 2012; KANDEMIR; ACUR, 2012; JAIN; ADIL; ANANTHAKUMAR, 2013; GRANT et. al, 2013), posicionamento estratégico e gestão de custos (QUESADO; RODRIGUES, 2007; CAVALVANTI; FERREIRA; ARAUJO, 2013) e estratégias de produção e gestão de custos (PACHECO 2010; PACHECO; CALARGE, 2012; FULLERTON; KENNEDY; WIDENER, 2013).

Além desta introdução, o estudo apresenta o referencial teórico acerca dos temas mencionados, seguido da descrição dos procedimentos metodológicos adotados na pesquisa. Na sequência apresenta-se o capítulo de análise dos dados, finalizando com as conclusões e as referências utilizadas na construção do estudo.

\section{Referencial Teórico}

\subsection{Posicionamento estratégico}

Porter (1979) define estratégia empresarial como processo de ações agressivas e defensivas, a fim de ganhar uma posição sustentável no mercado para enfrentar, com sucesso, as cinco forças competitivas. São elas: 1) concorrentes na indústria; 2) entrantes potenciais; 3 ) substitutos; 4) compradores; 5) fornecedores. Neste cenário, para obter vantagem competitiva, Porter (1989) descreveu três estratégias genéricas para competição, a saber: 1) estratégia de liderança de custo, 2) estratégia de diferenciação, e 3) estratégia de foco. Estes conceitos representam diferentes orientações estratégicas que a empresa deve buscar, a fim de realizar seus objetivos.

A estratégia de liderança de custo é um conjunto integrado de medidas tomadas para produzir bens ou serviços, com características que são aceitáveis para os clientes, com o 
menor custo em relação aos concorrentes (GUAN; REHME; NORD, 2012). Além da produção em escala, os esforços para redução e controle de custos em todas as áreas da empresa são importantes nesta estratégia (SUMER; BAYRAKTAR, 2012).

A estratégia de diferenciação envolve a criação de uma posição de mercado, que é percebida como diferenciada, e que seja sustentável em longo prazo (BLANCHARD; GAIGNÉ; MATHIEU, 2012; MAKADOC; ROSS, 2013). Segundo Liu e Zhang (2013), a adoção da estratégia de diferenciação pode ser uma forma eficaz para que as empresas busquem nichos rentáveis em um mercado competitivo, e é predominante para produtos como bens de consumo.

$\mathrm{Na}$ estratégia de foco, as empresas podem concentrar a atuação em um determinado grupo de clientes ou em determinada área geográfica. Posteriormente a esta definição, as organizações podem optar pela atuação nas estratégias de liderança em custo ou diferenciação (MASCARENHAS, 2013). Observa-se, desta forma, que as empresas focadas podem se especializar em uma destas dimensões e buscar obter vantagens competitivas sobre seus concorrentes.

Existem autores (ACQUAAH; ARDEKANI, 2008; BAROTO; ABDULLAH; WAN, 2012; SUMER; BAYRAKTAR, 2012) que defendem a utilização de uma nova estratégia, chamada de híbrida. Esta estratégia é definida como a utilização das estratégias de custos e diferenciação de forma integrada. Sumer e Bayraktar (2012) propõem uma nova estrutura para as estratégias genéricas de Porter, com o quarto nível estratégico, a saber: 1) estratégia de liderança de custo, 2) estratégia de diferenciação 3) estratégia de foco, 4) e estratégia híbrida.

Sumer e Bayraktar (2012) explicam que a estratégia híbrida não se trata de perda de foco, ou a empresa sem estratégia, criticada por Porter. Os autores exemplificam que uma empresa pode optar pela competição por custo de seus produtos e buscar diferenciação nos serviços prestados, ou seja, optar por uma estratégia (custo) e buscar características de outra (diferenciação) para obter melhor desempenho.

Acquaah e Ardekani (2008) justificam que as empresas que buscam uma estratégia competitiva híbrida poderão atingir melhor desempenho do que as empresas que adotarem uma estratégia singular. Os autores destacam que a implementação de uma estratégia competitiva integrada resulta em aumento da vantagem competitiva.

Baroto, Abdullah e Wan (2012) citam os exemplos da Toyota e Walmart como empresas que adotaram estratégias híbridas. No caso da Toyota, a empresa buscou competir na estratégia de liderança de custos; porém, desenvolveu internamente um processo de produção que a possibilitou fabricar automóveis com nível de qualidade e variabilidade de modelos superior a seus concorrentes. Quanto à rede americana Walmart, percebe-se que o objetivo declarado da política de preços da empresa é totalmente focado na estratégia de liderança de custo; porém, a empresa busca diferenciação nos serviços oferecidos aos seus clientes. Para exemplificar, Baroto, Abdullah e Wan (2012) mencionam que a Walmart foi pioneira no lançamento dos cartões fidelidade, proporcionando descontos aos clientes que efetuam compras frequentes nas lojas da rede, e também na adoção da estratégia de vendas pela internet.

Pricop (2013) destaca que, independente da estratégia competitiva adotada pela empresa (liderança de custos, diferenciação, foco ou híbrida), o desempenho será mais satisfatório se essas estratégias forem planejadas de forma integrada entre todos os departamentos da organização, inclusive a manufatura.

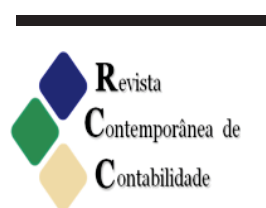




\subsection{Estratégias de manufatura}

Estratégia de manufatura pode ser entendida como a definição de políticas e planos para a utilização de recursos produtivos de uma empresa, visando ao alcance e à sustentação de sua estratégia competitiva (ORTEGA; VEGA; MACHUCA, 2012; GRANT et al., 2013). Neste sentido, a vantagem competitiva pode ser alcançada de forma mais concisa, se a direção estratégica global do negócio for apoiada pela estratégia de manufatura (HAN; PORTERFIELD; LI, 2012), e se a estratégia de manufatura for definida baseada na estratégia de negócios (KANDEMIR; ACUR, 2012). Isso também é corroborado por outros autores (DUPONT, 2011; JAIN; ADIL; ANANTHAKUMAR, 2013).

Thun (2008) também destaca que a definição da estratégia de manufatura deve ser baseada na coordenação entre a estratégia de negócios e os recursos produtivos disponíveis nas empresas, e define esta prática como estratégia integrada de manufatura. A Figura 1 apresenta a metodologia da definição da estratégia integrada de manufatura:

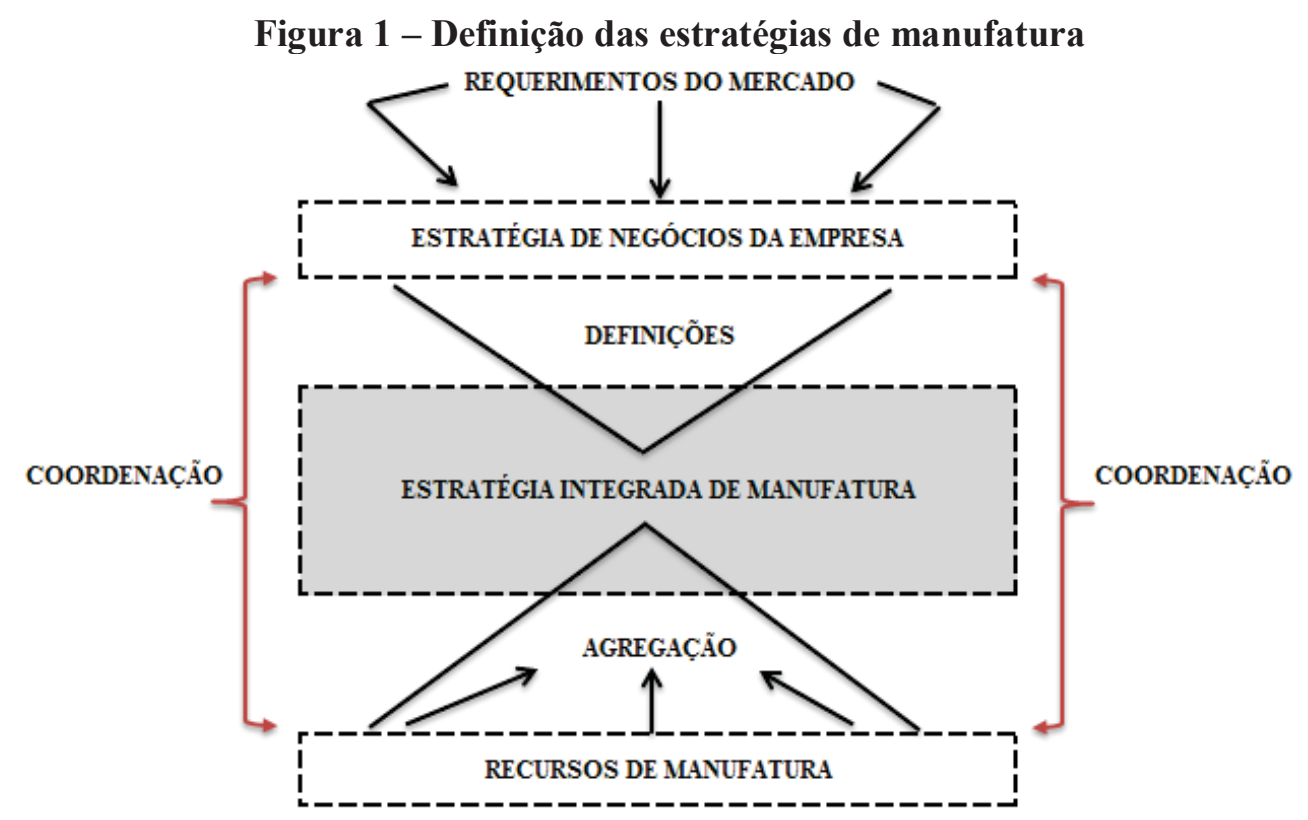

Fonte: Adaptado de Thun (2008).

Conforme mostrado na Figura 1, a empresa deve definir a estratégia de negócios com base nos requerimentos do mercado. Com esta definição deve-se analisar como os recursos produtivos disponíveis podem contribuir para obtenção da vantagem competitiva. Posteriormente a estas análises deve-se planejar a estratégia de manufatura buscando atender as necessidades do mercado e maximizar o desempenho de todos os recursos existentes na estrutura de produção da organização.

O trabalho seminal de Skinner (1969) introduziu a necessidade do processo de manufatura ocupar um papel proativo na busca pelos objetivos estratégicos dos negócios, e destaca que essa definição tem um caráter sistêmico, no sentido de apontar para uma abordagem global para gestão da estrutura produtiva. A Figura 2 apresenta o processo de determinação das estratégias de manufatura sugerido pelo autor.

Skinner (1969) propõe, inicialmente, a análise de mercado e da concorrência, e então, posteriormente, o estudo dos recursos produtivos disponíveis na empresa. Desta forma será 
possível planejar como a empresa poderá competir. Após um planejamento global considerando mercado (oportunidade e ameaças) e recursos (pontos fracos e pontos fortes), devem ser definidas as políticas de manufatura, que servirão de guia para a gestão da produção. Skinner (1969) propõe, ainda, que este processo seja controlado e reavaliado constantemente, com o intuito de se fazerem os ajustes e correções necessários, de acordo com as mudanças de mercado. Ao analisar a proposta de Skinner (1969) é possível observar o ciclo PDCA (planejar, executar, controlar e agir nas correções e ajustes).

Figura 2 - Processo de determinação das estratégias de manufatura

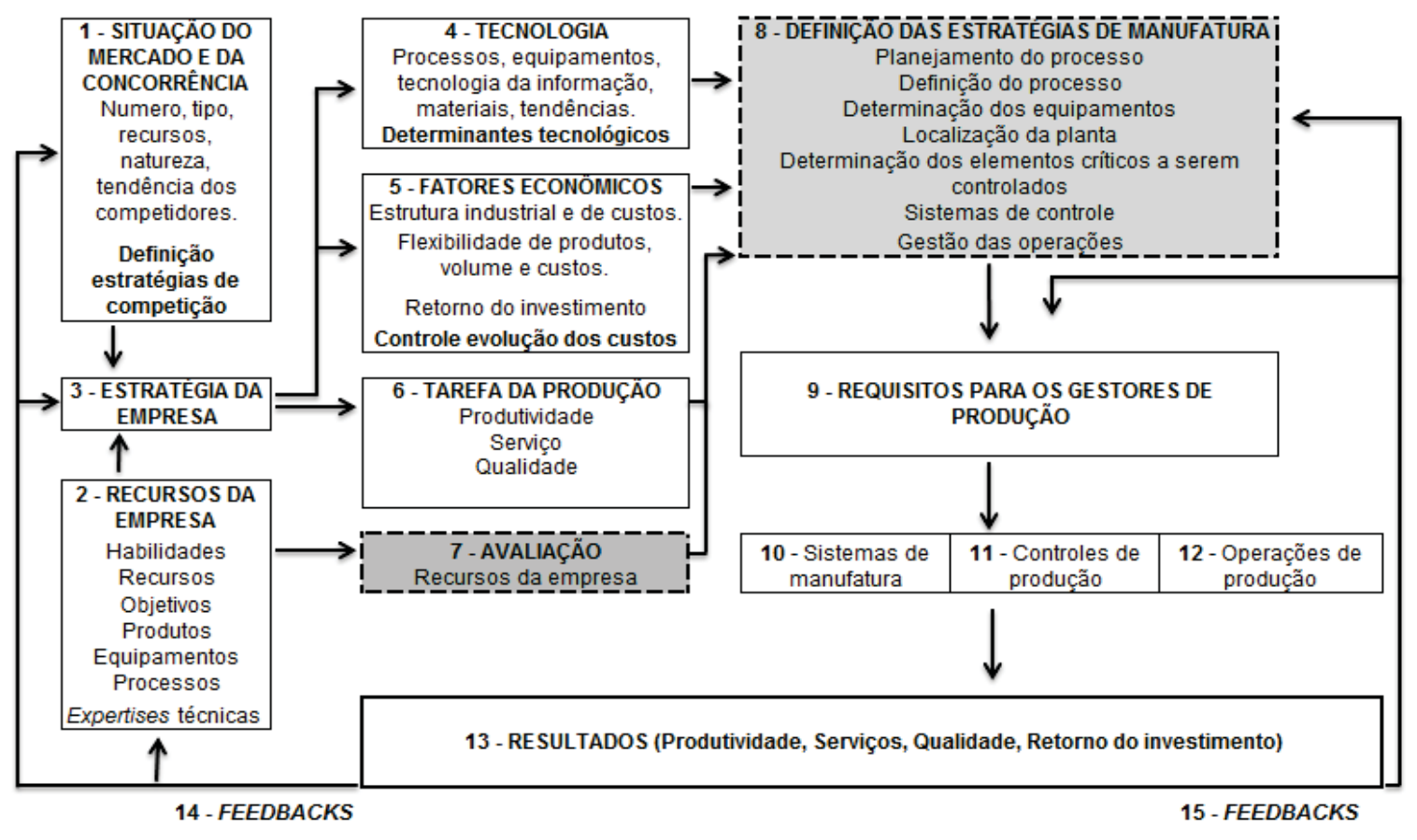

Fonte: Skinner (1969).

O processo de planejamento das estratégias de manufatura foi aprofundado por Skinner (1974), no sentido de propor que a função da manufatura é buscar a melhor forma de auxiliar a empresa a competir no mercado. Neste contexto o autor propôs um modelo de produção, chamado de fábricas focalizadas, no qual a empresa poderia organizar a produção em unidades de manufatura ou mini fábricas, de acordo com os produtos fabricados. Hill (2008) também aborda este tema e destaca que as estratégias de liderança de custos, diferenciação, foco ou híbridas exigem diferentes arranjos produtivos para melhorar a o desempenho da manufatura.

Pacheco (2010) destaca que a indústria automobilística tem apresentado contribuições importantes para a evolução das estratégias de manufatura, desde a linha de montagem desenvolvida pela Ford, até o sistema Toyota de produção (STP). De acordo com Fleury e Fleury (2008), a indústria automobilística utiliza, também, outras estratégias conhecidas como condomínios industriais e consórcios modulares, nos quais os fornecedores de componentes e peças instalam suas unidades manufatureiras na mesma área de produção do fabricante do produto final. O Quadro 1 sintetiza as principais estratégias de manufatura utilizadas e suas

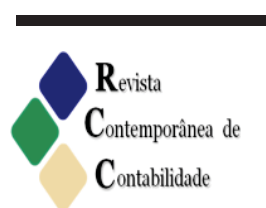


características. Também expõe com qual ênfase estratégica, cada estratégia de manufatura é mais alinhada.

Quadro 1 - Estratégia de manufatura indicada para cada ênfase estratégica

\begin{tabular}{|l|c|c|}
\hline \multicolumn{1}{|c|}{ Estratégia de manufatura } & $\begin{array}{c}\text { Características do mercado de } \\
\text { atuação }\end{array}$ & Ênfase estratégica \\
\hline Sistema de produção Fordista & $\begin{array}{c}\text { Alta demanda de produção, baixa } \\
\text { variabilidade de produtos }\end{array}$ & Liderança de custo \\
\hline $\begin{array}{l}\text { Sistema de produção enxuta } \\
\text { (STP) }\end{array}$ & $\begin{array}{c}\text { Baixa demanda de produção, alta } \\
\text { variabilidade de produtos }\end{array}$ & Diferenciação, Híbrida \\
\hline Fábricas Focalizadas & $\begin{array}{c}\text { Baixa e média demanda de produção, } \\
\text { alta e média variabilidade de produtos }\end{array}$ & $\begin{array}{c}\text { Liderança de custos, } \\
\text { Diferenciação, Híbrida }\end{array}$ \\
\hline $\begin{array}{l}\text { Condomínios industriais, } \\
\text { Consórcios modulares }\end{array}$ & $\begin{array}{c}\text { Baixa demanda de produção, alta } \\
\text { variabilidade de produtos, produtos de } \\
\text { alto valor agregado }\end{array}$ & Diferenciação, Híbrida \\
\hline
\end{tabular}

Fonte: Adaptado de Pacheco (2010).

No Quadro 1 são mostradas as estratégias de manufatura mais utilizadas recentemente pelas empresas (PACHECO, 2010). Tais estratégias servem como alternativas na busca de eficiência e diferencial competitivo. A estratégia de liderança de custos requer sistemas produtivos mais preparados para produção em escala, já que esta característica influencia consideravelmente no custo dos produtos. A estratégia de fábricas focalizadas é mais indicada para empresas que atuam em mercado de baixa e média demanda e alta variabilidade de produção. A adoção de estratégias de diferenciação ou híbridas requer sistemas de produção flexíveis e preparados para atender mercados de baixa demanda e alta variabilidade de produtos.

Para Miltenburg (2008), o planejamento da estratégia de manufatura deve buscar a melhoria na qualidade, entrega e a redução e controle dos custos dos produtos fabricados. Pacheco e Calarge (2012) não abordam somente redução de custos, mas gestão de custos, e destacam, também, que esta gestão de custos pode ser utilizada de forma coordenada com a estratégia da manufatura, dentro da visão de médio e longo prazo.

\subsection{Gestão estratégica de custos}

Shank (1989) destaca a necessidade da transição da análise de custo gerencial para a gestão estratégica de custos, e argumenta que as informações contábeis internas devem apoiar o desenvolvimento e a implementação da estratégia de negócio. Nessa visão, de acordo com o autor, a gestão de negócios é um ciclo contínuo de quatro estágios de processos: 1) formulação de estratégias; 2) comunicação da estratégia para toda a organização; 3) desenvolvimento e execução de táticas para implementar as estratégias; 4) desenvolvimento e implementação de controles para monitorar o sucesso das etapas de implementação e, consequentemente, o sucesso na realização dos objetivos estratégicos. As informações de custo desempenham papel importante em cada um desses estágios.

Shank e Govindarajan (1995) citam que o surgimento de gestão estratégica de custos é o resultado da coordenação de três temas subjacentes que são retirados da literatura. Os três temas são: 1) análise da cadeia de valor; 2) análise do posicionamento estratégico; 3) e análise 
dos direcionadores de custos. Shank (1989) também destacou que, com a definição da estratégia de negócios, a empresa deve organizar seus métodos de gestão de custos, alinhando os sistemas de controles e indicadores com esta ênfase estratégica.

Conforme Ashvine e Shafabi (2011), novas abordagens de gestão de negócios estão sendo implementadas nas organizações, tais como: gestão da qualidade total, reengenharia de processos, melhoria contínua, entre outras. Estas filosofias requerem organizações ágeis e flexíveis, fornecendo valor agregado a produtos e serviços para os clientes, com preços competitivos. Com esta mudança no ambiente de negócios das organizações tem-se observado o crescimento da utilização de uma nova abordagem contábil, chamada de gestão estratégica de custos.

Ashvine e Shafabi (2011) argumentam, também, que gestão estratégica de custos é uma filosofia de melhoria de custos e receitas, não somente gestão de custos, também gestão de receitas. Além disso, ainda busca melhorar a produtividade, maximizar o lucro, e melhorar a satisfação dos clientes. Esta filosofia desempenha um papel importante na determinação do futuro da companhia, porque promove a continuidade, encontrando maneiras para ajudar o gestor a tomar a decisão certa para criar valor ao cliente. Nesta perspectiva, Abdel e Mclellan (2013) citam que a gestão estratégica de custos representa uma atitude proativa, em que todos os custos de produtos e serviços resultam da decisão da gestão da empresa. Quanto às técnicas, os autores definem que é um conjunto de instrumentos que podem ser utilizados individualmente para dar suporte a objetivos específicos; ou juntos, para servir às necessidades globais da organização.

Segundo Tontiset e Choojan (2012), ao longo das últimas décadas, as pressões competitivas em todo o mundo, o crescimento da indústria e avanços em tecnologia e manufatura tem mudado a economia, e estão fazendo com que muitas empresas mudem a maneira como elas operam. Esta mudança na competição de negócios tem afetado profundamente a contabilidade de custos e a gestão de custos. Esta visão também foi corroborada por Quesado e Rodrigues (2007), que enfatizam as mudanças ocorridas no comportamento dos consumidores e argumentam que, para sobreviver neste ambiente cada vez mais dinâmico e competitivo, é fundamental o foco na gestão estratégica de custos, que deverá ser encarada como um processo de mudança em termos estruturais, comportamentais e culturais, na procura da melhoria contínua e na criação de valor para o cliente.

O custeio meta como uma das ferramentas da gestão estratégica de custos é o sistema que apoia o processo de redução de custos na fase de design e desenvolvimento de um novo produto, enquanto o custeio kaizen é o sistema que dá suporte para o processo de redução de custos na fase de manufatura de produtos já existentes; ou seja, refere-se a contínuas acumulações de pequenas atividades de melhorias, ao invés de melhoria inovadora (ZENGIM; ADA, 2010; PACHECO, 2010).

\subsection{Estudos relacionados}

O Quadro 2 apresenta estudos empíricos recentes presentes na literatura, que utilizam a gestão estratégica de custos, estratégias de manufatura e posicionamento estratégico, destacando os objetivos dos trabalhos e os principais achados relacionados aos estudos. As pesquisas referem-se ao período de 2010 a 2013, tanto no âmbito nacional quanto internacional.

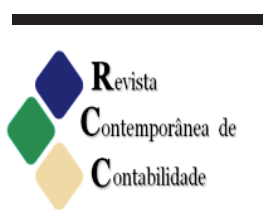


Posicionamento estratégico, estratégias de manufatura e gestão de custos: estudo de caso em uma empresa ...

Quadro 2 - Estudos Relacionados sobre posicionamento estratégico, gestão de custos e estratégia de manufatura

\begin{tabular}{|c|c|c|}
\hline Autor/ano & Objetivos & Principais achados \\
\hline Pacheco (2010) & $\begin{array}{l}\text { Propor um modelo de } \\
\text { gestão sistêmica de } \\
\text { custos para ser utilizada } \\
\text { conjuntamente com a } \\
\text { estratégia de manufatura. }\end{array}$ & $\begin{array}{l}\text {-As estratégias de manufaturas e a gestão de custos passaram a } \\
\text { ser importantes instrumentos de melhorias para as condições } \\
\text { de competitividade; } \\
\text {-A aplicação do modelo integrado às estratégias de } \\
\text { manufatura possibilita, às empresas, uma estrutura enxuta e } \\
\text { eficiente. }\end{array}$ \\
\hline $\begin{array}{l}\text { Tontiset; } \\
\text { Choojan (2012) }\end{array}$ & $\begin{array}{l}\text { Examinar o efeito do } \\
\text { sucesso da gestão } \\
\text { estratégica de custos no } \\
\text { desempenho r das } \\
\text { empresas de fabricação } \\
\text { de eletrônicos na } \\
\text { Tailândia. }\end{array}$ & $\begin{array}{l}\text {-A gestão estratégica de custos influencia positivamente no } \\
\text { desempenho da empresa; } \\
\text {-Tem influência positiva na tomada de decisão, planejamento } \\
\text { e controle da eficácia e apoio à diretoria; } \\
\text {-O envolvimento dos empregados influencia positivamente o } \\
\text { sucesso da gestão estratégica de custo. }\end{array}$ \\
\hline $\begin{array}{l}\text { Almeida; } \\
\text { Machado; } \\
\text { Panhoca (2012) }\end{array}$ & $\begin{array}{l}\text { Investigar as práticas de } \\
\text { gestão de custos nas } \\
\text { empresas da indústria da } \\
\text { construção ruver civil } \\
\text { paranaense, que seguem } \\
\text { a tipologia das } \\
\text { estratégias de Porter. }\end{array}$ & $\begin{array}{l}\text { As empresas que atuam na estratégia de liderança de custos } \\
\text { atribuem maior importância a práticas de gestão de custos, tais } \\
\text { como: controles gerenciais com foco em controle de custos, } \\
\text { metas de operação específicas, orçamentos e controles } \\
\text { orçamentários rígidos. }\end{array}$ \\
\hline $\begin{array}{l}\text { Pacheco; } \\
\text { Calarge (2012) }\end{array}$ & 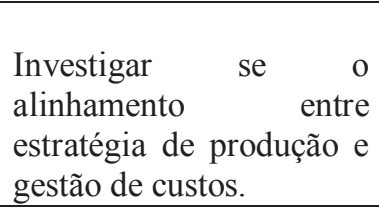 & $\begin{array}{l}\text {-É necessário incluir a cadeia de valor nas políticas } \\
\text { estratégicas; } \\
\text {-A gestão de custos pode ser utilizada para apoiar o } \\
\text { alinhamento entre estratégias de produção e políticas } \\
\text { organizacionais. }\end{array}$ \\
\hline $\begin{array}{l}\text { Cavalcanti; } \\
\text { Ferreira; Araujo } \\
(2013)\end{array}$ & $\begin{array}{l}\text { Investigar a integração } \\
\text { existente entre o } \\
\text { posicionamento } \\
\text { estratégico e a gestão de } \\
\text { custos de uma empresa } \\
\text { beneficiadora de aço } \\
\text { inox. }\end{array}$ & $\begin{array}{l}\text { - A empresa utiliza a estratégia de diferenciação; } \\
\text { - Os custos elevados com manutenção de estoques e custo de } \\
\text { ociosidade da produção não são considerados; } \\
\text { - O gerenciamento da cadeia de suprimentos é inconsistente; } \\
\text { - Não existe um sistema de custos que possibilite a análise; } \\
\text { - A gestão de custos adotada pela empresa não está coerente } \\
\text { com o seu posicionamento estratégico. }\end{array}$ \\
\hline $\begin{array}{l}\text { Fullerton; } \\
\text { Kennedy; } \\
\text { Widener (2013) }\end{array}$ & $\begin{array}{l}\text { Verificar como a gestão } \\
\text { de custos pode contribuir } \\
\text { para a implementação da } \\
\text { estratégia de manufatura } \\
\text { enxuta. }\end{array}$ & $\begin{array}{l}\text { Foram identificadas práticas que podem apoiar a } \\
\text { implementação da manufatura enxuta: Implementação de um } \\
\text { sistema simplificado de divulgação de informações de custos, } \\
\text { fluxo de valor de custeio, implementação de um sistema de } \\
\text { indicadores de desempenho baseado em custos, treinamento } \\
\text { dos funcionários sobre práticas de gestão de custos. }\end{array}$ \\
\hline
\end{tabular}

Fonte: Elaborado pelos autores.

O Quadro 2, mostra estudos que reforçam a importância da gestão estratégica de custos. Também destacam que a gestão de custos pode proporcionar apoio para formulação de estratégias e definição de políticas organizacionais.

\section{Metodologia da Pesquisa}

O presente trabalho apresenta uma pesquisa aplicada com abordagem qualitativa, pois os fatos foram observados na empresa estudada sem qualquer interferência por parte dos pesquisadores. No que se refere aos objetivos, a pesquisa é descritiva, pois visa a facilitar o 
entendimento do assunto abordado por meio de descrição das características que envolvem o caso estudado.

Quanto aos procedimentos técnicos, trata-se de um estudo de caso único. Este procedimento investiga fenômenos detalhadamente, pois o estudo de caso destaca a atualidade do contexto através de eventos ou condições e seus relacionamentos, visando ao entendimento deste fenômeno por meio de discussão dos resultados, interpretações e comparações (YIN, 2005).

O processo de coleta de dados foi efetuado por meio de entrevistas, análise documental e observação direta na empresa objeto de estudo. A empresa é uma multinacional com sede na Alemanha, foi fundada em 1926 e atua no segmento metal mecânico, mais especificamente na fabricação de produtos destinados aos mercados agropecuário, de jardinagem e florestal. Para atender ao mercado global, conta com unidades produtivas na Alemanha, EUA, Áustria, Suíça, China e Brasil. A unidade no Brasil está localizada no estado do Rio Grande do Sul, na cidade de São Leopoldo, e conta, atualmente, com um quadro de aproximadamente 2.000 funcionários. Os respondentes da pesquisa foram o gerente do departamento de melhoria contínua, funcionário da empresa há 8 anos; o supervisor de produção, que é funcionário da empresa há 5 anos; e o coordenador de controladoria, que é funcionário da empresa há 28 anos.

As entrevistas foram conduzidas de forma espontânea, a partir de um roteiro prédefinido pelos pesquisadores. Na entrevista, conforme Yin (2005), o entrevistador pode tanto indagar, dos respondentes-chave, os fatos relacionados a um assunto, quanto pedir a opinião deles sobre determinados eventos.

Os documentos analisados referem-se a relatórios gerenciais de controle, tais como: dados relativos à redução de custos obtidos com a implementação de projetos de melhoria contínua já em vigor e cronograma de implantação de projetos que estão em andamento, informativo de produtividade, controle de retrabalhos, controle de entregas de pedidos e informações relacionadas a treinamento das equipes de produção.

Seguindo as orientações de Yin (2005), foi elaborado o protocolo do estudo de caso, que compreendeu as principais fases da pesquisa de campo. O objetivo deste protocolo é definir a maneira como o trabalho será conduzido, assim como a data das visitas, tempo estimado e os recursos empregados para coletar as informações necessárias à realização do estudo. Este envolve as seguintes etapas principais: Programação da data e hora de visita, locais da empresa a serem visitados, pessoas a serem entrevistadas e procedimentos na empresa para coleta de dados (entrevistas, observações do processo e análise de documentos gerenciais).

As entrevistas foram conduzidas com o objetivo de identificar qual a estratégia competitiva da empresa (custo, diferenciação, foco ou híbrido), qual a estratégia de manufatura adotada, e como a gestão de custos pode apoiar a integração entre o posicionamento estratégico definido com a estratégia de manufatura adotada pela organização. Também se buscou identificar se a empresa possui programas de melhoria contínua que contemplem projetos de redução de custos, e se estes projetos são mensurados. Por fim, buscou-se identificar qual o método de custeio gerencial adotado pela organização, se é efetuada a análise dos preços praticados pela concorrência, e se as informações gerenciais de custos são utilizadas para tomada de decisões estratégicas. Ainda se buscou verificar se a empresa utiliza o custo meta e custo kaizen, se existem diferentes controles de custos para cada linha de produção, e se é efetuada a avaliação de resultados destas linhas separadamente.

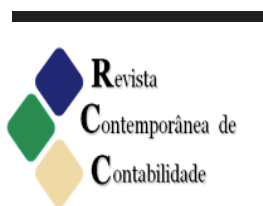


A etapa de análise de dados consistiu em examinar e classificar os dados coletados nas visitas, análise documental e observação direta na empresa. Primeiramente, efetuou-se a análise das respostas recebidas durante as entrevistas realizadas, bem como dos registros efetuados durante as visitas na empresa. Posteriormente, fez-se a análise dos documentos observados (listados anteriormente), no intuito de descrever características que possam ser consideradas importantes e necessárias para alcançar o objetivo de pesquisa apresentada no trabalho. Desta forma é possível efetuar a apresentação dos resultados obtidos com o estudo e as conclusões acerca do tema.

\section{Apresentação e Análise dos Dados}

\subsection{Caracterização geral da empresa}

Conforme descrito anteriormente, a empresa estudada é uma multinacional com sede na Alemanha, fundada em 1926 e que atua no segmento metalmecânico, especificamente na fabricação de produtos destinado aos mercados agropecuário, de jardinagem e florestal. Atualmente a organização conta com aproximadamente 11 mil colaboradores em todo o mundo, está presente em mais de 160 países e tem seus produtos comercializados por mais 38.000 pontos de vendas. No Brasil, os principais produtos fabricados e comercializados pela empresa são: motosserras, roçadeiras e aparadores elétricos, com uma produção aproximada de 50.000 unidades mensais. A empresa também produz cilindros para motores, fabricando aproximadamente 500.000 unidades por mês. No mercado brasileiro, a empresa possui mais de 2.400 pontos de vendas.

\subsection{Resultados}

Quanto à produção de cilindros para motores, identificou-se que a empresa compete na estratégia de liderança de custos, pois a produção é de alto volume e estes produtos são destinados tanto para produção dos motores dos equipamentos produzidos internamente (motosserras, roçadeiras e aparadores elétricos), quanto para outras empresas que utilizam este produto no mercado interno e exterior.

Nos mercados agropecuário, de jardinagem e florestal foi identificado que a empresa compete na estratégia de diferenciação. Trata-se de produtos com valor agregado, que depois de produzidos são comercializados diretamente com os consumidores através de lojas próprias. Esta estratégia é justificada devido às características deste produto e seu manuseio, que exige alto treinamento e especialização técnica por parte dos vendedores. Também é efetuado treinamento específico quanto à utilização do produto no ato da venda com cada consumidor. Além das lojas próprias, atualmente, no Brasil, a organização comercializa somente para uma rede de varejo. Neste caso, os vendedores desta rede participam de treinamentos na empresa para entender as características e propriedades de cada tipo de produto. Desta forma constatou-se, também, que a empresa apresenta características de diferenciação nos serviços de venda, pois as lojas próprias que são chamadas de concessionárias de vendas também fazem o serviço de assistência técnica e entrega técnica (treinamento para utilização do produto no ato da venda). 
Conforme identificado nas entrevistas, existe uma forte competição pelo menor custo entre as próprias unidades produtivas do grupo localizadas em diversos países (Alemanha, Brasil, EUA, Áustria, Suíça e China) no momento do lançamento de novos produtos. Quando estes produtos são desenvolvidos, é efetuado um orçamento coordenado pela matriz na Alemanha, e a empresa do grupo que apresentar o melhor custo do produto terá maiores possibilidades de ser a responsável pela produção do mesmo. Este procedimento sugere que a empresa possui características da estratégia híbrida, já que, no mercado, compete por diferenciação, porém, entre as unidades do grupo, também compete por custo. Com base no posicionamento estratégico e na concorrência entre as unidades da organização, a empresa planejou e adequou sua estratégia de manufatura.

A empresa começou a adotar práticas de manufatura enxuta na década de 1980. A partir de 2006, a organização optou em estender esta estratégia para todas as linhas de produção do grupo. Na unidade brasileira, os trabalhos iniciaram com a implementação de um projeto piloto na linha de produção de motores. Após aproximadamente um ano de treinamento, aplicações e ajustes, as mensurações de desempenho relativas a antes versus depois da implantação da estratégia de manufatura enxuta (ME) apontaram os seguintes resultados (Tabela 1):

Tabela 1 - Comparativa dos dados da linha piloto (motores)

\begin{tabular}{lcc}
\hline \multicolumn{1}{c}{ Descrição dos dados } & Antes ME & Depois ME \\
\hline Produção mensal da linha de manufatura & 52.000 unidades & 62.000 unidades \\
Número de operadores da linha de manufatura & 60 operadores & 55 operadores \\
Turnos de trabalho diário na linha de manufatura & 3 turnos & 2 turnos \\
\hline
\end{tabular}

Fonte: Dados da pesquisa.

Conforme se pode observar na Tabela 1, após a implementação da manufatura enxuta, a empresa obteve benefícios com aumento de produção e redução de custos, tais como: aumento da produtividade, redução de custos com mão de obra e redução de turnos de trabalho. Posteriormente a este teste piloto, foi implementada a estratégia de manufatura enxuta na produção de cilindros para motores. Os resultados obtidos na linha de produção de cilindros corroboram os benefícios observados na primeira implementação (linha de motores) e são apresentados na Tabela 2:

Tabela 2 - Comparativa de dados na linha de cilindros para motores

\begin{tabular}{lcc}
\hline \multicolumn{1}{c}{ Descrição dos dados } & Antes ME & Depois ME \\
\hline Produção mensal da linha de manufatura & 500.000 unidades & 500.000 unidades \\
Número de operadores da linha de manufatura & 110 operadores & 92 operadores \\
Turnos de trabalho diário na linha de manufatura & 3 turnos & 2 turnos \\
\hline Fonte: Dados da pesquisa. & &
\end{tabular}

Observa-se que a produção mensal não teve alteração, já que este não era objetivo da empresa, pois este aumento de unidades produzidas poderia gerar estoques desnecessários, aumentando os custos da organização. No entanto, obteve-se redução de mão de obra direta, com a diminuição de 110 para 92 o número de operadores, e redução de 3 para 2 turnos de trabalho. As análises destes resultados foram consideradas satisfatórias e suportaram a implementação da estratégia de produção nas demais linhas de manufatura.

Atualmente, a unidade brasileira conta com a estratégia de produção enxuta implantada em todas as linhas de manufatura da empresa. Este sistema é suportado pelo

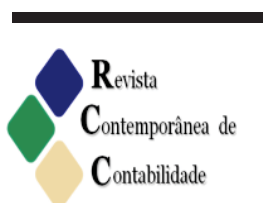

ISSN 2175-8069, UFSC, Florianópolis, v. 13, n. 28, p. 81-98, jan./abr.2016 
kaizen (melhorias contínuas), em que praticamente todos os projetos implantados contemplam redução de desperdícios e, consequentemente, redução de custos. A meta para o retorno sobre o investimento (quando necessário) varia de 1 a 2 anos, em cada projeto implantado. No que se refere à forma de mensuração, na maioria dos projetos são avaliados em como impactam positivamente no ganho da empresa e geram prêmios ao(s) funcionário(s) autor(es) da ideia, com base nesta medição. No entanto, também são realizadas melhorias contínuas que não são mensuradas financeiramente, mas podem apresentar outros benefícios, como melhoria nas condições de trabalho, saúde, meio ambiente e ergonomia, por exemplo.

Os entrevistados também destacaram que são realizados treinamentos constantemente com os colaboradores da empresa, e a partir destes treinamentos são montados os times orientados à redução de desperdícios (chamados de TORD), com os seguintes procedimentos sobre métodos e ferramentas da estratégia de manufatura enxuta.

- Treinamento (conceitos, exemplos, aplicação);

- Avaliação e definições de kaizen que podem ser aplicados;

- Formação dos grupos de melhoria contínua;

- Delegação da responsabilidade dos projetos de kaizen por equipes;

- Definição de ferramentas a ser implementadas (estudo dos projetos);

- Implantação de projeto piloto;

- Extensão do projeto a outras linhas de produção da empresa;

- Padronização do processo.

Percebe-se que a estrutura de manufatura foi organizada alinhada com o posicionamento estratégico da empresa. Além da produção enxuta, que foi implementada em todo o processo produtivo, a empresa também montou mini fábricas que produzem motosserras, roçadeiras e aparadores elétricos separadamente. No que se refere à produção de cilindros, devido às características diferentes (alto volume e baixo custo), também foi montada uma estrutura produtiva específica.

Quanto ao sistema de custeio, foi identificado, na entrevista, que para fins fiscais utiliza-se o sistema de custeio por absorção/integral, tendo em vista que a empresa é tributada pelo lucro real. Para fins gerenciais é utilizado o conceito do custeio por absorção, com alguns ajustes que foram efetuados internamente, como depreciações com vidas mais curtas e a depreciação dos itens não cessam após o final da vida útil do mesmo; porém, neste caso, são depreciados $50 \%$ da taxa normal. Também são adicionados juros sobre ativo imobilizado nos respectivos centros de custos, para alocação indireta nos produtos.

A empresa faz comparações de preços com os concorrentes através das listas de preços existentes nas lojas. Quanto ao custo, as informações são utilizadas para formação de preços de venda e para estabelecer comparativo e concorrência entre empresas do grupo. As informações de custo são utilizadas de maneira estratégica como, por exemplo, produzir determinado produto na unidade com menor custo.

Quanto à utilização do custeio meta e kaizen, tradicionalmente utiliza-se, como base para tomada de decisão, o custo padrão. Eventualmente, para alguns casos pontuais, como concorrência entre empresas do grupo, utiliza-se o custo meta. Quanto ao controle de custos, não existe rigidez maior entre as unidades de motores (estratégia de diferenciação) e cilindros (estratégias de custos). Também se verificou que a empresa não controla e analisa os resultados por cada linha de manufatura separadamente, mas da empresa como um todo, em cada planta produtiva. Os sistemas de custeio, tanto contábil fiscal como o gerencial, são 
totalmente integrados no ERP da empresa, através do software SAP, o qual oferece a possibilidade de calcular o custo padrão e fazer a análise das variações ocorridas. Este mesmo software também possibilita calcular o custo meta. A partir da definição dos parâmetros são feitas simulações, até definir a estrutura adequada para produzir com o custo desejado.

\subsection{Discussão dos resultados}

No mercado agropecuário, de jardinagem e florestal verificou-se que a empresa compete na estratégia de diferenciação, tanto na produção quanto na prestação de serviços. Também apresenta características da estratégia híbrida, pois existe competição pelo menor custo entre as unidades do grupo, no lançamento de novos produtos. Identificou-se que, na produção de cilindros para motores, a empresa compete na estratégia de liderança de custos. Estes achados alinham-se com a pesquisa de Baroto, Abdula e Wan (2012), que apontaram características de estratégia híbrida na Walmart e na Toyota, e também com Tontiset e Choojan (2012), que concluíram que a gestão de custos apoia a tomada de decisões estratégicas, tal como em qual unidade de manufatura produzir determinados produtos para melhorar a rentabilidade da organização.

O modelo do sistema de produção desenvolvido pela Toyota serviu de base para implementação da estratégia de manufatura definida pela empresa. Esta estratégia foi definida coordenada com a estratégia de negócios da organização. Outra estratégia de manufatura adotada pela empresa foi a organização de fábricas focalizadas, propostas inicialmente por Skinner (1974). Neste sentido, a empresa organiza suas operações divididas em mini fábricas, nas quais se produzem motosserras, roçadeiras, aparadores elétricos em linhas diferentes, organizadas em layout que aumentem a eficiência da produção, de acordo com as características de cada produto.

A empresa utiliza a gestão de custos para fins contábeis e gerenciais: esta gestão dá suporte para análise do posicionamento estratégico e da estratégia de produção. Estes achados alinham-se com o trabalho de Pacheco (2010), que propôs um modelo de gestão de custos integrado a estratégia de manufatura, utilizando o custo meta e kaizen, como principais ferramentas. Também se verificou que praticamente todos os projetos de melhoria contínua são baseados na redução de custos. Este achado alinha-se com as conclusões de Fullerton, Kenedy e Widener (2013), que dentre as práticas de gestão de custos que podem apoiar a estratégia de manufatura destaca-se a implementação de sistema de indicadores de desempenho baseado em custos; e com os apontamentos de Pacheco e Calarge (2012), de que a gestão de custos pode ser utilizada para alinhar a estratégia de manufatura às políticas organizacionais das empresas.

No que se refere ao controle de custos, não se identificaram diferenças quanto à rigidez no controle entre as unidades de motosserras, roçadeiras e aparadores elétricos (estratégia de diferenciação), e cilindros para motores (estratégia de liderança de custos). Estes achados são diferentes dos resultados apresentados por Almeida, Machado e Panhoca (2012), que concluíram que as empresas que atuam na estratégia de liderança de custos efetuam maior controle sobre seus custos do que empresas que atuam na estratégia de diferenciação.

Por meio da análise dos dados obtidos, entende-se que a empresa planejou o processo de gestão de custos alinhado com o seu posicionamento estratégico. Esta conclusão diverge dos resultados apresentados por Cavalcanti, Ferreira e Araujo (2013), em estudo desenvolvido

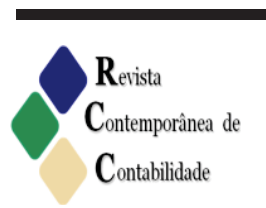

ISSN 2175-8069, UFSC, Florianópolis, v. 13, n. 28, p. 81-98, jan./abr.2016 
em uma empresa beneficiadora de aço inox, que atua na estratégia de diferenciação. Os autores concluíram que a organização não desenvolveu um sistema de custos que possibilite análise do alinhamento entre posicionamento estratégico e a gestão de custos, além da gestão de custos adotada pela empresa não estar coerente com o seu posicionamento estratégico.

Os resultados apresentados sugerem que a empresa planejou a sua estratégia de manufatura com base no posicionamento estratégico definido pela organização, e que a gestão de custos é utilizada de forma estratégica para controlar as operações, e para fornecer informações que apoiem a tomada de decisões na organização, além de buscar e manter a vantagem competitiva.

\section{Considerações Finais}

Este trabalho teve como objetivo analisar como a gestão de custos apoia a integração entre posicionamento estratégico e estratégia de manufatura em uma subsidiária brasileira de uma empresa alemã, que atua no segmento metalmecânico. Os achados do artigo indicam que a empresa adota estratégia de liderança de custos na linha de cilindros para motores, e estratégia de diferenciação na sua principal linha de produtos (motosserras, roçadeiras e aparadores elétricos). Também se identificou que, no segmento de produtos em que a empresa compete na estratégia de diferenciação, existe uma forte concorrência por custos entre as unidades do grupo, o que sugere características de estratégia híbrida.

Entende-se que a gestão de custos é utilizada para apoiar a integração entre o posicionamento estratégico e estratégias de manufatura, por meio da utilização das informações geradas pelos métodos de custeio contábil e gerencial, visto que estes fornecem informações que dão suporte à tomada de decisões na organização. Esta situação foi confirmada, uma vez que foi constatada a utilização de custeio por absorção para fins fiscais, custeio padrão, custeio meta e custeio kaizen, para fins gerenciais. Ainda se verificou que as estratégias de manufatura enxuta e fábricas focalizadas foram adotadas para atender necessidades de diferenciação de produtos. A estratégia de manufatura enxuta é suportada pelas melhorias contínuas (kaizen) na produção, e praticamente todos os projetos são baseados em redução de custos. Foi identificado pela pesquisa que a empresa efetua avaliação de resultados mensalmente, contemplando, de forma global, toda a unidade do Brasil. Entende-se que a focalização da produção por linha do produto permite, à empresa, uma avaliação de resultados segregados por unidade produtiva, o que permitiria melhor controle gerencial e fortaleceria ainda mais a utilização da gestão de custos como apoio à integração entre posicionamento estratégico e estratégias de manufatura.

Para futuras pesquisas sugere-se analisar a influência destas ações no resultado financeiro da empresa, visto que esta foi uma das limitações do estudo. Também se sugere aprofundar a discussão da integração entre posicionamento estratégico, estratégia de produção e gestão de custos em empresas que atuem em outros segmentos. 


\section{Referências}

ABDEL, Al Sherine; MCLELLAN, John. Strategy and management accounting practices alignment and its effect on organizational performance. Journal of Accounting, Business \& Management, v. 20, n. 1, p. 1-27, 2013.

ACQUAAH, Moses; ARDEKANI, Masoud. Does the implementation of a combination competitive strategy yield incremental performance benefits? a new perspective from a transition economy in Sub-Saharan Africa. Journal of Business Research, v. 61, n. 4, p. 346$354,2008$.

ALMEIDA, Lauro Brito; MACHADO, Esmael Almeida; PANHOCA, Luiz. Práticas de gestão de custos e perspectivas estratégicas: um estudo na indústria da construção do estado do Paraná. Revista Brasileira de Gestão de Negócios, v. 14, n. 44, p. 353-368, 2012.

ASHVINE, Kumar; SHAFABI, Khanpur. Strategic cost management - suggested framework for 21 st century. Journal of Business and Retail Management Research, v. 5, n. 2, p. 118 $130,2011$.

BAROTO, Mas Bambang; ABDULLAH, Muhammad Madi Bin; WAN, Hooi Lai. Hybrid strategy: a new strategy for competitive advantage. International Journal of Business and Management, v. 7, n. 20, p. 120-133, 2012.

BLANCHARD, Pierre; GAIGNÉ, Carl; MATHIEU, Claude. Trade costs and international strategy of firms: the role of endogenous product differentiation. Regional Science and Urban Economics, v. 42, n. 1, p. 1023-1036, 2012.

CAVALCANTI, Maria Aparecida do Nascimento; FERREIRA, Helem Mara Confessor; ARAUJO, Aneide Oliveira. Análise do posicionamento estratégico para implementação da gestão estratégica de custos: um estudo de caso em uma empresa do setor de beneficiamento de aço inoxidável. Revista Ambiente Contábil, v. 5, n. 1, p. 75-92, 2013.

DUPONT, André Cardoso. Proposição de um método para concepção da estratégia de produção: uma abordagem a partir dos conceitos de subunidades estratégicas de negócios. Dissertação (Mestrado em Engenharia de Produção e Sistemas), Programa de Pósgraduação em Engenharia de Produção e Sistemas, Universidade do Vale do Rio dos Sinos, 2011.

FLEURY, Afonso Carlos Corrêa; FLEURY, Maria Tereza Leme. Estratégias Empresariais e Formação de Competência: um quebra-cabeça caleidoscópico da indústria brasileira, $3^{\mathrm{a}}$ ed. São Paulo: Atlas, 2008.

FULLERTON, Rosemary; KENNEDY, Frances; WIDENER, Sally. Management accounting and control practices in a lean manufacturing environment. Accounting, Organizations and Society, v. 38, n. 1, p. 50-71, 2013. 
GRANT, Noeleen, CADDEN, Trevor, MCIVOR, Ronan, HUMPHREYS, Paul. A taxonomy of manufacturing strategies in manufacturing companies in Ireland. Journal of Manufacturing Technology Management, v. 24, n. 4, p. 488-510, 2013.

GUAN, Wei; REHME, Jakob; NORD, Tomas. Classification and retail positioning into strategic groups the case of DIY retailers and builders merchants in Sweden. International Journal of Retail \& Distribution Management, v. 40, n. 8, p. 570-591, 2012.

HAN, Chaodong; PORTERFIELD, Tobin; LI, Xiaolin. Impact of industry competition on contract manufacturing: an empirical study of US manufacturers. International Journal of Production Economics, v. 138, n. 1, p. 159-169, 2012.

HILL, Alex. How to organise operations: focusing or splitting? International Journal of Production Economics, v. 112, n. 2, p. 646-654, 2008.

JAIN, Bhurchand; ADIL, Gajendra; ANANTHAKUMAR, Usha. An instrument to measure factors of strategic manufacturing effectiveness based on Hayes and Wheelwright's model. Journal of Manufacturing Technology Management, v. 24, n. 6, p. 824-829, 2013.

KANDEMIR, Destan; ACUR, Nuran. Examining proactive strategic decision-making flexibility in new product development. Journal of Product Innovation Management, v. 29, n. 4, p. 608-622, 2012.

LIU, Qian; ZHANG, Dan. Dynamic pricing competition with strategic customers under vertical product differentiation. Management Science, v. 59, n. 1, p. 84-101, 2013.

MAKADOK, Richard; ROSS, David Gaddis. Taking industry structuring seriously: a strategic perspective on product differentiation. Strategic Management Journal, v, 34, n. 1, p. 509-532, 2013.

MASCARENHAS, Briance. The industry-focused international strategy. Management International Review, v. 53, n. 2, p. 251-267, 2013.

MILTENBURG, John. Setting manufacturing strategy for a factory-within-afactory. International Journal of Production Economics, v. 113, n. 1, p. 307-323, 2008.

ORTEGA, Cesar; VEGA, Pedro; MACHUCA, Jose Antonio Dominguez. Analysis of interaction fit between manufacturing strategy and technology management and its impact on performance. International Journal of Operations \& Production Management, v. 32, n. 8, p. 958-981, 2012.

PACHECO, Milton Gomes. Modelo de gestão sistêmica de custos integrado a estratégia de manufatura. Tese (Doutorado em Engenharia de Produção), Programa de Pós-graduação em Engenharia de Produção, Universidade Metodista de Piracicaba, 2010. 
PACHECO, Milton Gomes; CALARGE, Felipe Araújo. Gestão de custos no modelo de integração da estratégia de manufatura. Revista Transinformação, v. 17, n. 1, p. 61-77, 2012 .

PORTER, Michael. How competitive forces shape strategy. Harvard Business Review, v. 57, p. 137-145, 1979.

PORTER, Michael. Vantagem competitiva: criando e sustentando um desempenho superior. Rio de Janeiro: Campus, 1989.

PRICOP, Oliver Constantin. Critical implications of the economic concepts for the strategic management, Journal Metalurgia International, v. 18, n. 4, p. 116-121, 2013.

QUESADO, Patricia Rodrigues; RODRIGUES, Lúcia Lima. A gestão estratégica de custos em grandes empresas portuguesas. Revista Iberoamericana de Contabilidad de Gestión, v. 5, n. 10, p. 121-143, 2007.

SHANK, John; GOVINDARAJAN, Vijay. Gestão estratégica de custos - a nova ferramenta para a vantagem competitiva. Rio de Janeiro: Campus, 1995.

SHANK, John. Strategic cost management: new wine, or just new bottles. Journal of Management Accounting Research , v. 1, n. 1, p. 47-65, 1989.

SKINNER, Wickham. Manufacturing-missing link in corporate strategy. Harvard Business Review, v. 47, p. 136-145, 1969.

SKINNER, Wickham. The focused factory. Harvard Business Review, v. 52, p. 113-121, 1974.

SUMER, Keren; BAYRAKTAR, Cahit Ali. Business strategies and gaps in Porter's typology: a literature review. Journal of Management Research, v. 4, n. 3, p. 100-119, 2012.

THUN, J. H. Empirical analysis of manufacturing strategy implementation. International Journal of Production Economics, v. 113, n. 1, p. 370-382, 2008.

TONTISET, Nattawu; CHOOJAN, Jirapan. Successful of strategic cost management of electronics manufacturing business in Thailand: an empirical research of its antecedents and consequences. International Journal of Business Strategy, v. 12, n. 3, p. 133-149, 2012.

YARBROUGH, Larry; MORGAN, Neil; VORHIES, Douglas. The impact of product market strategy-organizational culture fit on business performance. Journal of the Academy of Marketing Science, n. 39, v. 4, p. 555-573, 2011.

YIN, Robert K. Estudo de caso planejamento e métodos. 3 ed. São Paulo: Bookman, 2005. ZENGIM, Yasemin; ADA, Erhan. Cost management through product design: target costing approach. International Journal of Production Research, n. 48, v. 19, p. 5593-5611, 2010. 